\title{
Peak ground acceleration produced by local earthquakes in volcanic areas of Campi Flegrei and Mt. Vesuvius
}

\author{
Danilo Galluzzo, Edoardo Del Pezzo, Mario La Rocca and Simona Petrosino \\ Osservatorio Vesuviano, Istituto Nazionale di Geofisica e Vulcanologia, Napoli, Italy
}

\begin{abstract}
The scaling law of the seismic spectrum experimentally calculated at Mt. Vesuvius and Campi Flegrei is used to constrain the estimate of the maximum expected peak acceleration of ground motion. The scaling law was calculated for earthquakes recorded at BKE and OVO stations in the period 1997-2000 for Mt. Vesuvius and for earthquakes occurred during the 1983-1984 bradyseismic crises at Campi Flegrei. For Mt. Vesuvius the scaling law clearly deviates from a constant stress drop relation in the whole range of magnitude $\left(0.4<M_{D}<3.6\right)$ whilst constant stress-drop is found for Campi Flegrei data $\left(0.7<M_{D}<3.4\right)$. These results are used to give a first estimate of the maximum ground motion corresponding to the largest magnitude $\left(M_{\max }\right)$ inferred in the two investigated areas by the Gutenberg-Richter formula. The values of the seismic moment $M_{0}$ and the characteristic source radius corresponding to $M_{\max }$ are used to evaluate the peak ground acceleration PGA. This parameter is determined by stochastic simulation of ground motion. Two different methods (Random Vibration Theory (RVT) (Boore, 2003) and ground motion generated from a Gaussian distribution with $\sigma=a_{\mathrm{ms}}$ (GMG)) give slightly different values of PGA. The values of PGA were $0.10 \mathrm{~g}$ (RVT), $0.14 \mathrm{~g}$ (GMG) for Mt. Vesuvius and $0.04 \mathrm{~g}$ (RVT and GMG) for Campi Flegrei.
\end{abstract}

Key words peak ground acceleration - scaling law - stochastic method-Mt. Vesuvius - Campi Flegrei

\section{Introduction}

In the estimate of seismic risk, the determination of ground motion parameters like spectral characteristics, peak ground displacement and peak ground acceleration is very important for a quantitative assessment of the problem. The knowledge of these parameters provides the basis for a classification of the territory and identifies the areas for which great damage in

Mailing address: Dr. Danilo Galluzzo, Osservatorio Vesuviano, Istituto Nazionale di Geofisica c Vulcanologia, Napoli, Via Diocleziano 328, 80124 Napoli, Italy; e-mail: galluzzo@ov.ingv.it case of strong earthquakes is expected. The determination of ground motion parameters can be carried on by numerically simulating the time history related to the maximum expected earthquake in a given area. Among the techniques used for the estimate of ground motion parameters, the stochastic method (Boore, 2003 ) is widely used to predict the ground motion due to a seismic input, which can be modeled taking into account source, path and site effects. The method provides the ground motion by combining a parametric description of amplitude spectrum with a random phase spectrum. This technique has been applied to estimate peak parameters of ground motion in many regions of the world (Akinci et al., 2001; Castro et al., 2001 Atkinson et. al., 2002) and is particularly useful to simulate the ground motion for the frequency range usually investigated by earthquake engineering. 
The most critical step in the application of the stochastic method is the correct definition of the source spectrum characteristics (shape and amplitude). This requires knowledge of the source model and scaling law of the seismic spectrum. The scaling law relates the seismic moment $M_{0}$ to the linear dimension of the source (Lay and Wallace, 1995, 373-379). For a constant stress drop, the moment scales with the characteristic rupture dimension as

$$
M_{0} \propto r^{3} .
$$

Consequently, as $\mathrm{r}$ is proportional to the inverse corner frequency and $M_{0}$ to the flat portion of the log-log far field displacement seismic spectrum, the level of the flat portion of the seismic spectrum scales with the cube of the inverse corner frequency. In the last three decades the observed scaling laws have been used to verify the scale invariance of the earthquake source in a wide range of Magnitude for both tectonic and volcanic areas. Scaling invariance was studied first by Aki (1967). He investigated the relationship between $f_{c}$ and $M_{0}$ showing that $M_{0} \propto f_{c}^{-3}$, corresponding to a stress drop independent of magnitude. Later, Geller (1976) used a data set of moderate and large earthquakes to derive the scaling rules for fault parameters.

More recent studies have shown that for smaller earthquakes (Magnitude 3) $M_{0}$ is not proportional to $f_{c}^{-3}$ (Archuleta et al., 1982): the seismic spectrum shows the same corner frequency for low values of the seismic moment and the stress-drop decreases with magnitude.

To explain these observations, a cut-off frequency, $f_{\max }$, was introduced to reproduce the steep fall-off at high frequency (Hanks, 1979, 1982), but a serious debate arose about the origin of $f_{\max }$, if it is site or source generated (Anderson and Hough, 1984). More recently, source heterogeneity has been introduced through barrier/asperity models to explain the anomalous behavior of the scaling law at low Magnitude. For these models the simple spectral shape is modified by including the "patch» corner frequency corresponding to the asperity characteristic size (Papageorgiou, 2003).
Aki (1986) proposed the hypothesis that self-similarity holds within individual ranges of seismic moments. Recently Abercrombie (1995) demonstrates using borehole data that the apparent breakdown of self-similarity is due to the attenuation in the upper few kilometers of the Earth's crust. Conversely Hiramatsu et al. (2002) show that the break down of relationship $M_{0} \propto f_{c}^{-3}$ is an artifact caused by a too low sampling rate of the data.

Although the knowledge of the scaling laws correctly defines the source spectrum, other factors must be taken into account for an effective prediction of ground motion. The ability of the stochastic method to predict ground response increases when site and path effects are included in the numerical simulation. The path effects include both the distance-dependence of the amplitude spectrum and the attenuation, which can be modeled introducing the geometrical spreading coefficient and the quality factor $Q$. Site effects generally depend on the local geology and could produce an amplification of the seismic spectrum, which should be taken into account in the numerical simulation of ground motion.

A careful modeling of source, path and site effects yields ground motion simulations very close to the real ground response, allowing a reliable estimate of ground motion peak parameters.

For the whole Italian territory hazard maps based on the expected PGA values are already available (www.mi.ingv.it; Slejko et al., 1998). In these maps the maximum expected acceleration for the volcanic areas of Mt. Vesuvius and Campi Flegrei is estimated considering the ground motion produced by strong tectonic earthquakes occurring in the Apennines. However, Mt. Vesuvius and Campi Flegrei are potentially very dangerous volcanoes and local volcano-tectonic earthquakes are expected in case of seismic crises which could precede a possible eruption. Thus, the possible occurrence of local earthquakes needs to be taken into account for a more detailed determination of the PGA in the Neapolitan volcanic areas. In these highly urbanized and densely populated areas possible severe damage could be produced by relative- 
ly small magnitude events occurring close to or inside the urbanized area.

In the present paper we estimate the PGA from ground motion simulation for potentially damaging local earthquakes at Mt. Vesuvius and Campi Flegrei. We first present a review of the experimental results on the scaling law of the seismic spectrum found at Mt. Vesuvius and Campi Flegrei with the aim of putting experimentally evaluated constraints for the predictions of the ground motion generated by local earthquakes. These two volcanic areas show apparently different patterns of Moment vs. corner frequency for the same range of magnitudes. The scaling law of the seismic spectrum for Mt. Vesuvius shows no constant stress drop in the whole range of magnitude $\left(0.4<M_{D}<3.6\right)$ whilst Campi Flegrei data reveal constant stressdrop in the range $\left(0.7<M_{D}<3.4\right)$. This information is then coupled with the largest magnitude value expected for both the areas, allowing us to evaluate peak ground acceleration by means of stochastic simulations.

\section{Campi Flegrei: data set, method, analysis of data and results}

Campi Flegrei is a $12 \mathrm{~km}$ diameter caldera located close to the city of Naples in Southern Italy. This volcanic zone is the site of periodic uplift episodes (bradyseism), which are accompanied by seismic crises. The two most recent bradyseismic crises occurred in 1969-1972 and 1982-1984. During the latter bradyseismic episode a ground uplift of $1.8 \mathrm{~m}$ centered in Pozzuoli town (fig. la) was accompanied by more than 16000 shallow earthquakes located in Pozzuoli-Solfatara area $\left(0.1<M_{D}<4.2\right)$ (Aster et al., 1992). The overall time pattern of the uplift episodes is reported in fig. 1b. The same figure shows that since 1985 there were also short duration uplift episodes, the major of which (ground uplift of about $7 \mathrm{~cm}$ ) occurred in the spring 1989 . The last seismic crises occurred on July 2-7 and August 22, 2000. In these periods two small earthquake swarms occurred during an uplift of about $4 \mathrm{~cm}$, which interrupted the descending trend started in 1985 (Saccorotti et al., 2001).

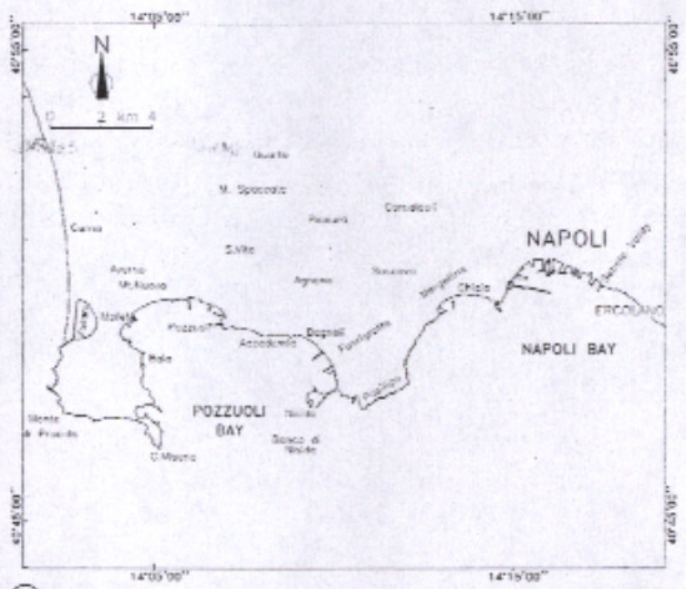

(a)

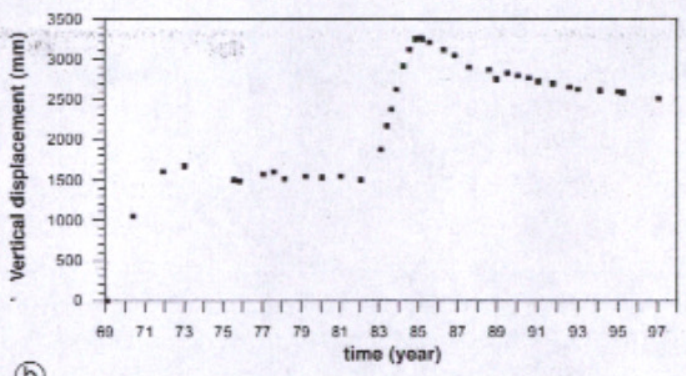

(b)

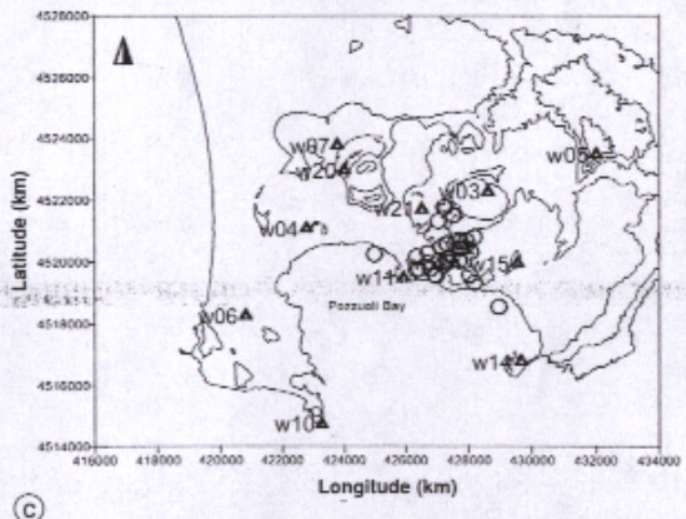

Fig. 1a-c. a) Map of Campi Flegrei Area. b) Vertical Ground Movement measured at Pozzuoli in the period 1969-1998. c) Epicenter locations (open circles) of 32 selected events used for the source parameter analysis. Open triangles indicate digital stations of University of Wisconsin (redrawn from $P 1$ ). 


\subsection{Data set}

Most of the seismic data available at Campi Flegrei belong to the swarms which occurred in the period 1983-1984. Seismic source scaling was investigated at Campi Flegrei by Del Pezzo et al. (1987), hereafter referred to as Pl. The epicenters of seismic events used by $\mathrm{P} 1$ are displayed in fig. 1c. Seismic signals were recorded by 12 three-component digital stations (dynamic range $=106 \mathrm{db}$ ) equipped with $1 \mathrm{~Hz} 3$-component short period sensors HS101, whose amplitude response curve is almost flat in the frequency band 1-25 Hz (Powell, 1983). Seismic signals were digitized at 100 sps and detected by an automatic trigger system. Further details of these instruments and trigger system are reported in P1. A collection of 181 three-component seismograms from 32 selected events was used by $P 1$ to estimate seismic source parameters.

\subsection{Review of method, analysis and results}

A detailed description of method and analysis of data is reported in $P 1$. Here only a brief sketch is reported. Spectra for $P$ and $S$ phases were calculated using the FFT algorithm applied to data in $1.28 \mathrm{~s}$ and $2.56 \mathrm{~s}$ windows, respectively for $P$-phase on the radial seismogram and $S$-phase on the transversal component of motion. Deconvolution for the amplitude response curve and integration were performed to obtain displacement spectra.

Spectral data were fit to the following formula (Boatwright, 1980):

$$
\log D(f)=\log \Omega_{0}+\pi f \frac{T}{Q}-0.5\left(1+\left(\frac{f}{f_{c}}\right)^{2 \gamma}\right)
$$

where $D(f)$ is the ground motion displacement spectra, $\gamma$ determines the high frequency spectrum decay, $Q$ is the average quality factor along the ray path and $T$ is the travel time, $\Omega_{0}$ is the low frequency spectral level and $f_{c}$ is the corner frequency. These parameters were determined by an iterative non-linear inversion of far field spectral data whose details are in $P 1$.
The iterative method was performed by fixing $Q$-value $(Q \in[120-300]$ in frequency range $1-16 \mathrm{~Hz}$ ) obtained by Del Pezzo et al. (1985) using the coda wave technique. These authors verified the hypothesis of S-wave composition for coda waves by estimating S-wave $Q\left(Q_{s}\right)$ from the slope of the spectrum flat portion. The estimated values of $Q_{S}(110 \pm 50)$ showed a good agreement with low frequency coda $Q . Q_{P}$ obtained by the iterative inversion $(P 1)$ was at least of a factor 2 different from $Q_{s}$.

Anyway, due to the short travel time, the value of $Q$ poorly affects the estimate of $\Omega_{0}$ and $f_{c}$.

Site effects were also estimated by Del Pezzo et al. (1993), hereafter referred to as $P 2$,
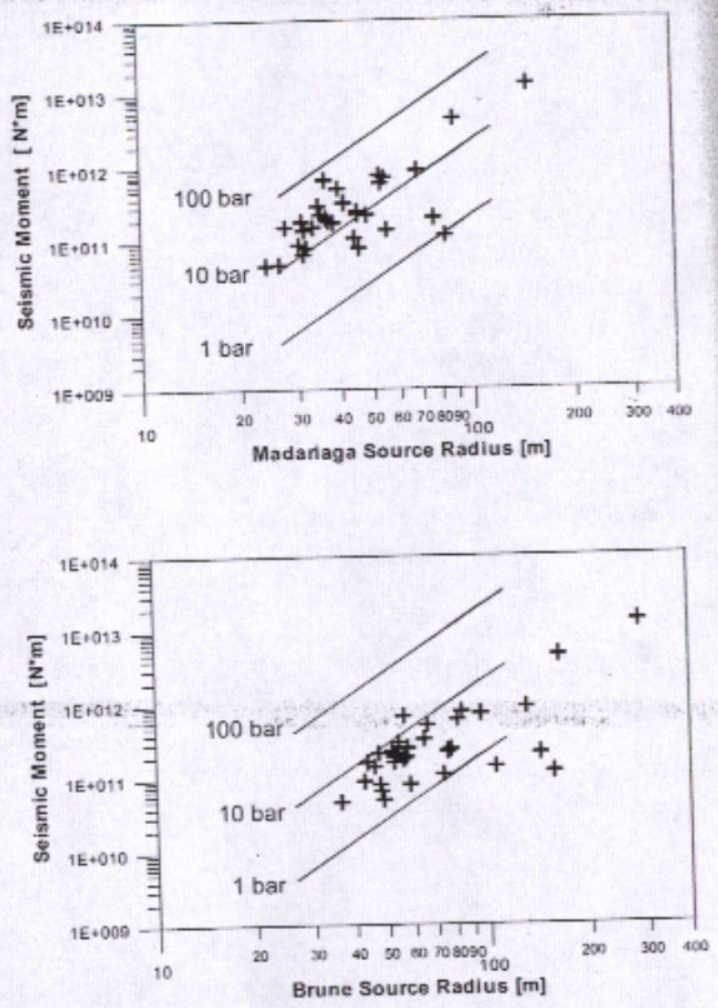

Fig. 2. Seismic moment versus Madariaga's source radius and Brune's source radius. Bars indicate standard deviation errors. Lines of equal stress drop (1, $10,100)$ are also displayed. 

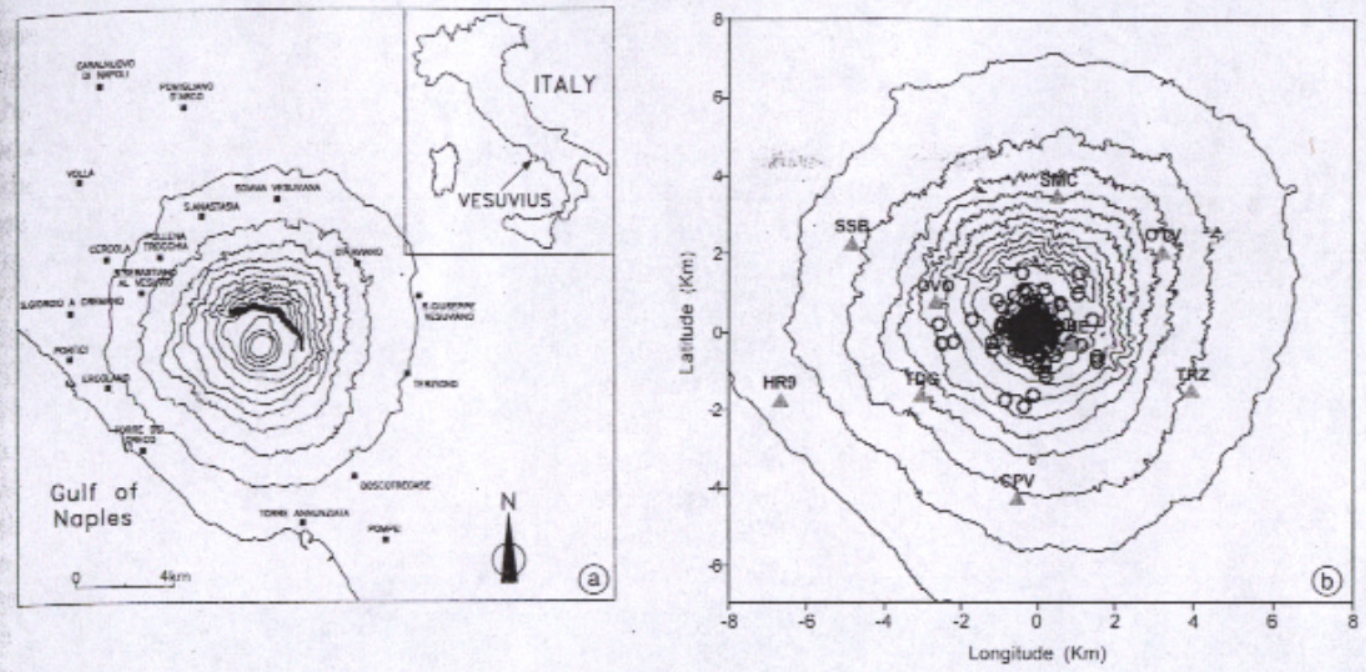

Fig. 3a,b. a) Map of Mt. Vesuvius area showing the topography (equidistance $=100 \mathrm{~m}$ ) and the largest surrounding towns. b) Map of epicenters occurred in 1999-2000 at Mt. Vesuvius. Solid triangles indicate the Osservatorio Vesuviano permanent network.
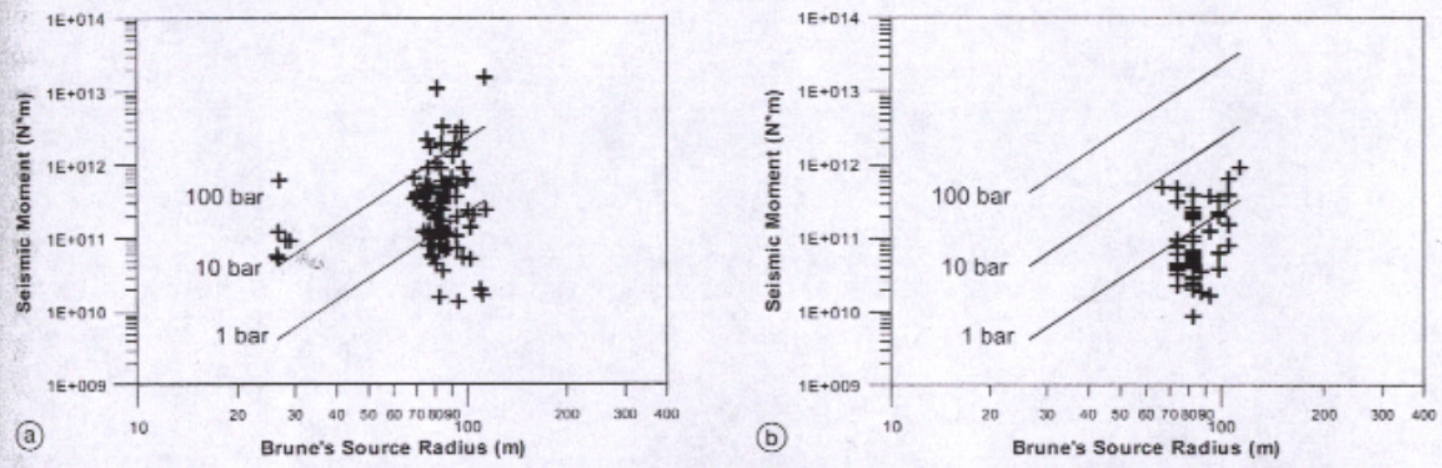

Fig. 4a,b. a) Seismic moment versus Brune's source radius for Mt. Vesuvius for data set recorded at BKE station. Lines of equal stress drop $(1,10,100)$ are also represented. b) The same for data set collected at OVO station.

who investigated how the site geology affects the seismic radiation spectrum at Campi Flegrei for a selected subset of the same data used in $P 1$, composed of 32 small earthquakes $(M<2)$ (fig. 2 in $P 2$ ). Seismic site response was determined using the method of the spectral ratios for $\mathrm{S}$ waves preliminary corrected for attenuation in the frequency range $1-15 \mathrm{~Hz}$ $(P 2)$. A reference station (W21), located at the center of the network, was chosen on the base of the highest number of recorded earthquakes. The spectral ratio for $\mathrm{S}$ waves was also estimated using as reference the «average» spectrum obtained for each seismic event by log averaging the seismic spectra over the stations (fig. 4a,b from $P 2$ ). Results show that the spectral pattern is approximately uniform in the range 1-6 Hz with amplification factor not 
greater than 2. Maximum site effects are in the range $8-15 \mathrm{~Hz}$ (a factor of 0.2 for $\mathrm{W} 10$ and W14 - see fig. 4 in $P 2$ ). As in the estimate of spectral parameters $(P 1)$, spectra were averaged over the stations (at least 4 stations), all the differences in the amplification level can be reasonably considerate smoothed. For this reason the measured spectral shape at Campi Flegrei can be reasonably considered unaffected by severe site effects. The seismic moment $M_{0}$ was calculated from $\Omega_{0}{ }^{P S}(P 1)\left(\Omega_{0}\right.$ calculated respectively for $\mathrm{P}$ and $\mathrm{S}$ waves) and by applying the relationship (Boatwrigth, 1980)

$$
\Omega_{0}^{p, s}=\frac{M_{0} Y_{0 \phi} F}{4 \pi \rho_{0} \alpha_{P, S}^{3} R} .
$$

In formula (2.2) $\alpha_{P, S}$ is the $\mathrm{P}$ or $\mathrm{S}$ wave velocity $\left(\alpha_{P}=3 \mathrm{~km} / \mathrm{s}\right.$ and $\left.\alpha_{S}=1.73 \mathrm{~km} / \mathrm{s}\right), \rho_{0}=2.2 \mathrm{~g} \cdot \mathrm{cm}^{-3}$ is the density, $Y_{\theta \phi}^{P}=0.4$ and $Y_{\theta \phi}^{s}=0.25$ are the radiation pattern terms, $F$ equals 2 is the free surface operator and $R$ is the hypocentral distance $(P 1)$. The characteristic source radius was calculated for both Brune (1970) and Madariaga (1976) models ( $P 1)$, using the equations

Madariaga (1976) $\left\{\begin{array}{r}r_{P}=0.32 \times \frac{v_{s}}{f_{C}^{p}} \\ r_{s}=0.21 \times \frac{v_{s}}{f_{C}^{s}}\end{array}\right.$

Brune (1970)

$$
r_{s}=0.37 \times \frac{v_{s}}{f_{c}^{s}} .
$$

Stress drops were finally derived using (KeilisBorok, 1959)

$$
\Delta_{\sigma}=C \times \frac{M_{0}}{r^{3}}
$$

where $C$ is a factor depending on the fault geometry and is equal to 0.44 for a circular source. In fig. 2 (redrawn from $P 1$ ) seismic moment versus Madariaga's and Brune's characteristic source radii are displayed. In fig. 2 Madariaga's source radius values are obtained by averaging between the values of $r_{P}$ and $r_{S H}$ obtained with equation $2.3 \mathrm{a}, \mathrm{b}$. The results showed a constant stress drop with values around 20 bar (Madariaga model) and 4 bar (Brune model). In the present paper we consid. er only the results obtained from the Brune model in order to compare them with those obtained at Mt. Vesuvius. A table showing the complete list of the results is reported in $\mathrm{Pl}$.

\section{Mt. Vesuvius: data set, method, analysis of data and results}

Somma Vesuvius is a composite central volcano located in the Campanian Plain near the city suburbs of Naples (fig. 3a). Its activity began about $300-500 \mathrm{ky}$ ago (Santacroce, 1983). It is formed by an ancient caldera (Mt. Somma) and by a younger cone (Mt. Vesuvius). The Somma edifice is composed of lava flows and minor scoria fall deposits. The Somma Caldera consists of ring-faults formed during plinian and subplinian eruptions (Andronico et al., 1995). The Caldera has an elliptic shape and in the northern part the walls reach a maximum height of about $280 \mathrm{~m}$. Caldera rims are covered by the products of post 1631 activity (Rosi et al., 1987). After the last plinian event (A.D. 79, «Pompeis enuption) the volcanism concentrated in the Somma Caldera and in the southwestern part of the volcano. Mt. Vesuvius is one of the most potentially dangerous volcanoes in the world. Its past eruptions caused much damage to the surrounding towns. The last eruption occurred in 1944. At present Mt. Vesuvius activity is characterized by a low level of seismicity (a few hundred microearthquakes per year) and by a poor fumarolic activity located inside the crateric rim. The last seismic crisis occurred in 1999. During this crisis the maximum Magnitude was 3.6. It was also the maximum Magnitude since the last 1944 eruption. At present the seismic activity is monitored by a permanent seismic network. This network is composed of 23 short period stations. For more details about network history and characteristics see Castellano et al. (2001).

\subsection{Data set}

Historical seismicity has been characterized by low to moderate activity. Periods of in- 
creased activity occurred in 1978, 1989-1990, 1995-1996 and in 1999. Seismicity is located around the crater area and hypocentral depths do not exceed $6 \mathrm{~km}$ b.s.l. (Bianco et al., 1999). Source parameters and scaling law of seismic spectrum at Mt. Vesuvius have been studied by Del Pezzo et al. (2003), hereafter referred to as $P 3$. This work is based on a selected set of data recorded at the digital station BKE (Lennartz. PCM 5800, 12 bit dynamical range equipped with $1 \mathrm{~Hz}$ sensor) in the period 1997-2000. Data selection was based on the rms threshold of hypocentral solution, that is equivalent to considering the earthquake records with best signal to noise ratio $(P 3)$. The selected data set was composed by 93 earthquakes $\left(0.4<M_{D} \leq 3.6\right)$. The locations of the seismic events collected in the period 1999-2000 with best signal to noise ratio are shown in fig. $3 b$.

\subsection{Review of method, analysis and results}

Source parameters for Mt. Vesuvius earthquakes were calculated using site and attenuation corrected displacement spectrum of direct $\mathrm{S}$ waves. Details of this procedure are reported in P3. In the following only a brief sketch of the main aspects of methodology used in $P 3$ is reported. Attenuation parameters and site factors for Mt. Vesuvius have been calculated by Bianco et al., 1999, hereafter referred to as $B 1$. In $B 1$ body wave quality factor for $S$ waves $\left(Q_{S}\right)$ and the site factor were calculated from the slope of displacement spectral shape using the FFT algorithm on $2.048 \mathrm{~s}$ selected time window whose starting point was fixed a few samples before the $S$-phase onset. A $2.5 \%$ cosine taper was used to taper time windows for $S$-wave and resulting spectra were divided by frequency to obtain displacement spectra (B1). The average spectra showed a bump around the corner frequency $(10-12 \mathrm{~Hz})$ for some stations and, for this reason, the analysis was performed by the authors in two frequency bands (1-3.5 Hz and 15-24 Hz) (B1). Quality factor $Q_{S}$ resulted equal to 67 and 59 respectively for low and high frequency bands and was considered almost independent of frequency $(B 1)$.
Site amplification values for BKE station were also calculated by $B 1$, resulting 0.3 and 0.4 respectively for low and high frequency bands excluding the band between 10 and 12 $\mathrm{Hz}$. In $P 3$ the authors obtained the scaling law of earthquakes recorded at BKE, after corrections for attenuation $(B 1)$ and considering BKE as unaffected by severe site effects. In $P 3$ spectral displacement amplitude was estimated for a $2.5 \mathrm{~s}$ time-window after the onset of $S$-wave using both the horizontal components of seismograms recorded at BKE station. These authors based the choice of a $2.5 \mathrm{~s}$ time length on considerations on the best compromise between stability of spectral estimate, calculation speed and minimization of radiation pattern effect. The corner frequency $f_{c}$ was calculated by the intersection of low frequency trend and high frequency spectral envelope $(P 3)$. Low frequency level was estimated by averaging spectral amplitude between $1 \mathrm{~Hz}$ and $10 \mathrm{~Hz}$ and high frequency trend was estimated with a linear fit from $13 \mathrm{~Hz}$ to $25 \mathrm{~Hz}(P 3)$. The spectral shapes were visually inspected to reject disturbed data $(P 3)$. These results are also reported in the present paper (fig. $4 a, b)$. The characteristic source radius was calculated from $f_{c}$ values using the ed. (2.3 c) of Brune (1970). As the procedures used by $P 3$ and $P 1$ respectively for Mt. Vesuvius and Campi Flegrei are slightly different, we check in the present paper the results obtained for Mt. Vesuvius applying to a small set of data the method used for Campi Flegrei data obtaining similar results.

Stress drop was determined using eq. (2.4) $(P 3)$. Figure $4 \mathrm{a}$ reports the pattern of seismic moment as a function of source linear dimension together with constant stress drop lines $(\Delta \sigma=1,10,100$ bars $)$. The maximum value of seismic moment $\left(\approx 10^{13} \mathrm{Nm}\right)$ refers to October 9,1999 event with $M_{D}=3.6$ and $\Delta \sigma \approx 100$ bars. In the present work we also apply the technique used in $(P 3)$ to a data set composed of 53 well located earthquakes collected at the analogical station OVO during 2001 by assuming the same amplification site values and attenuation factors used for BKE. The pattern of seismic moment as a function of source radius for these selected earthquakes is shown in fig. $4 \mathrm{~b}$ : the source radius is practically constant. This is a conse- 
quence of the fact that the corner frequency is constant and does not depend on the value of the seismic moment $M_{0}$. We exclude an effect due to a cut-off frequency $f_{\max }$ because no cutoff frequency below the antialiasing frequency $(25 \mathrm{~Hz})$ is observed in the amplitude spectra.

\section{Ground motion simulation}

We calculate in the present paper the Peak Ground Acceleration (PGA) for the largest expected magnitude earthquake at Campi Flegrei and $\mathrm{Mt}$. Vesuvius. We use two methods based on the stochastic simulation of ground motion.

The first method estimates PGA using Random Vibration Theory (RVT) (Boore, 2003). The RVT method is summarized in the following steps:

i) The shape of acceleration spectrum $A(f)$ is defined as a function of source parameters $\left(f_{c}\right.$, $M_{0}\left(M_{\max }\right)$ ), path $(\mathrm{Q})$, radiation pattern $\left(Y_{\theta \phi}\right)$, geometrical spreading $(\mathrm{R})$, free surface operator $(\mathrm{F})$ and medium parameters $\left(\alpha_{P, S}, \rho_{0}\right)$ (see table I); hereafter all these parameters are defined as $\left\{K_{i}\right\}$ parameters.

ii) The moments $m_{k}$ of the squared spectral amplitude of acceleration are evaluated to calculate the numbers $N_{\mathrm{z}, e}$ of zero crossing and extrema of time series (Boore, 2003). iii) The ratio (PGA/ $\left.a_{\text {rms }}\right)$ of PGA to the root mean square acceleration ( $\left.a_{\mathrm{rms}}\right)$ is calculated by using Cartwrigth and Longuet-Higgins equation (Cartwrigth and Longuet-Higgins, 1956);

iv) Parseval theorem is used to obtain the estimate of the rms acceleration $\left(a_{\mathrm{rms}}=m_{2}(A(f\right.$, $\left.\left\{K_{i}\right\}\right)$ ) in terms of the squared amplitude spectrum of ground motion $\left|A\left(f,\left\{K_{i}\right\}\right)\right|^{2}$.

v) PGA value is calculated by considering the product between the values of $\left(\mathrm{PGA} / a_{\text {rns }}\right)$ evaluated at point (iii) and $a_{\text {rms. }}$.

In the present paper we also use a second method which can be considered a slight modification of the first method, hereafter defined as GMG method. This provides an estimate of PGA in the following steps:

i) the shape of acceleration spectrum $A\left(f,\left\{K_{i}\right\}\right)$ is defined.

ii) Parseval theorem is used to calculate $a_{\text {rms }}$ following the equation (Boore, 1983):

$$
a_{\mathrm{ms}}=\sqrt{f_{c} \times \int_{0}^{+\infty}\left|A\left(f,\left\{K_{i}\right\}\right)\right|^{2} d f} .
$$

iii) Ground motion acceleration $A(t)$ is generated using a gaussian distribution with $\sigma$ equal to $a_{\text {rms. }}$

iv) $\mathrm{PGA}$ is evaluated by considering the maximum amplitude of $A(t)$.

Table I. Parameters used for the evaluation of PGA in the areas of Campi Flegrei and Mt. Vesuvius.

\begin{tabular}{ccccc}
\hline & Campi Flegrei & Source & Mt. Vesuvius & Source \\
\hline$a$ & $4.00 \pm 0.10$ & De Natale et al. $(1986)$ & $7.02 \pm 0.10$ & $P 3$ \\
$b$ & $0.92 \pm 0.04$ & De Natale et al. $(1986)$ & $1.71 \pm 0.05$ & $P 3$ \\
$M_{\max }$ & $4.3 \pm 0.2$ & - & $4.1 \pm 0.1$ & - \\
$M_{0}$ & $(6.5 \pm 1.5) \times 10^{13} \mathrm{Nm}$ & - & $(1.2 \pm 0.2) \times 10^{13} \mathrm{Nm}$ & - \\
$f_{c}$ & $2.8 \pm 0.2 \mathrm{~Hz}$ & - & $10 \pm 1 \mathrm{~Hz}$ & $P 3$ \\
$Q_{s}$ & 110 & $P 1$ & 62 & $B 1$ \\
$R$ & $2 \mathrm{~km}$ & Aster et al. $(1992)$ & $3 \mathrm{~km}$ & $P 3$ \\
$\rho_{0}$ & $2.2 \mathrm{~g} / \mathrm{cm}^{3}$ & $P 1$ & $2.5 \mathrm{~g} / \mathrm{cm}^{3}$ & Zollo et al. $(2002)$ \\
$\alpha_{\mathrm{s}}$ & $3.0 \mathrm{~km} / \mathrm{s}$ & $P 1$ & $2.4 \mathrm{~km} / \mathrm{s}$ & Lomax et al. $(2001)$ \\
$Y_{0 \phi}^{5}$ & 1 & - & 1 & - \\
$F$ & 2 & $P 1$ & 2 & $P 3$ \\
\hline
\end{tabular}


v) The averaged value of PGA is calculated by performing many simulations $(\mathrm{N}>50)$ (the iterative procedure is repeated from point (i) to point (iv)).

The definition of spectral ground acceleration $A\left(f,\left\{K_{i}\right\}\right)$ and the setting of parameters $\left\{K_{i}\right\}$ are the most important aspects of the two above described methods. The shape of $A\left(f,\left\{K_{i}\right\}\right)$ is obtained from the second derivative of eq. (2.1) and is equal to

$$
A\left(f,\left\{K_{i}\right\}\right)=\frac{\Omega_{0} \times f^{2}}{\sqrt{1+\left(\frac{f}{f_{c}}\right)^{2 \times \gamma}}} \exp \left(-\frac{\pi f T}{Q}\right)
$$

Spectral ground acceleration $A\left(f,\left\{K_{i}\right\}\right)$ is estimated in the present paper for the maximum expected magnitude $M_{\max }$ in the investigated areas for a given recurrence time period. As is well known, the number of earthquakes that occurred in a time period $\tau$ can be represented by Gutenberg-Richter relation (Lay and Wallace, 1995)

$$
\log N(M)=a-b M
$$

where $N$ is the number of earthquakes with magnitudes in a fixed range around magnitude $M, a$ and $b$ are parameters respectively related to scismicity level and earth heterogeneity (Scholz, 1968). Equation (4.3) can be used to calculate the expected maximum magnitude $M_{\max }$. If $a$ and $b$ are estimated for data in a time period $\tau$, the ratio $a / b$ gives the value of $M_{\max }$ expected in that period. It is remarkable that $M_{\max }$ can be underestimated if the data set is composed by smallsize carthquakes (Davison and Scholz, 1985).

For Campi Flegrei area $a$ and $b$ parameters of Gutenberg-Richter distribution were calculated by De Natale et al. (1986). The seismic catalogue used in that work starts form 1975 and ends in 1984, but it is mainly composed of seismic events related to the bradyseism episode of 1983-1984. As the seismicity of Campi Flegrei area was very low from 1984 to 2000 , in the present paper we consider the seismic catalogue complete for the period 1975-2000.

More than 10000 seismic events $\left(0<M_{D}<\right.$ $<4.2$ ) recorded at least by two stations were used by De Natale et al. (1986). A further selection was made by considering $M_{D}=0.6$ as the lowest magnitude threshold of completeness. a and b parameters were obtained using a least square evaluation on the selected data and are reported in table I. Using these values we estimate the value of $M_{\max }$ equal to $4.35 \pm 0.30$ for Campi Flegrei area, for a recurrence period of 25 years (1975-2000).

For Mt. Vesuvius a and b parameters were evaluated using the complete available catalogue (1972-2002) (P3). The authors, in P3, used a technique based on a weighted least square approach and considered the catalogue complete from $M_{D}>1.8$. Results are reported in table I. For Mt. Vesuvius $M_{\max }$ was $4.10 \pm 0.20$ for a recurrence period of 30 years. The seismic moment $M_{0}$ corresponding to $M_{\max }$ can be calculated using empirical relationship (Lay and Wallace, 1985). For Campi Flegrei the empirical formula relating Moment to Magnitude was determined in the present paper by operating a least square fit on the available data. It results that

$$
\log M_{0}=9.9+0.9 M_{D}
$$

with uncertainty of $1 \%$ on best fit parameters.

For Mt. Vesuvius, we estimated $M_{0}\left(M_{\max }\right)$ using the formula calculated by $P 3$ :

$$
\log M_{0}=9.8+0.8 M_{D}
$$

$P 3$ gives the uncertainty equal to $1 \%$ the on best fit parameters. The formulas (4.4) and (4.5) are comparable in the limit of the experimental errors. By using eq. (4.4) and eq. (4.5), $M_{0}\left(M_{\max }\right)$ is equal to $6.5 \cdot 10^{13} \mathrm{Nm}$ for Campi Flegrei and to $1.2 \cdot 10^{13} \mathrm{Nm}$ for Mt. Vesuvius (table I).

The corner frequency $f_{c}$ associated to the maximum expected magnitude $M_{\max }$ is evaluated using the Brune formula $(2.3 \mathrm{c})$, where the value of the source radius rc corresponding to $M_{0}\left(M_{\max }\right)$ is estimated extrapolating the scaling relationships (figs. 2 and 4 ). The values of $f_{c}\left(M_{\max }\right)$ for Campi Flegrei and Mt. Vesuvius are reported in table $\mathrm{I}$.

Attenuation parameters $Q$ are fixed equal to 110 for Campi Flegrei $(P 1)$ and 62 for Mt. Vesuvius (B1). 
Table II. PGA values calculated for Campi Flegrei and Mt. Vesuvius by RVT and GMG methods.

\begin{tabular}{ccc}
\hline & PGA Campi Flegrei & PGA Mt. Vesuvius \\
\hline RVT & $0.04 \mathrm{~g}$ & $0.10 \mathrm{~g}$ \\
GMG & $0.04 \pm 0.01 \mathrm{~g}$ & $0.14 \pm 0.05 \mathrm{~g}$ \\
\hline
\end{tabular}

Geometrical spreading factor $1 / \mathrm{R}$ is obtained by evaluating the average hypocentral distance between the mean of source locations and the nearest station site. For Campi Flegrei we consider R equal to $2 \mathrm{~km}$ and for Mt. Vesuvius equal to $3 \mathrm{~km}(P 3)$ these values being the minimum possible hypocentral distances. $\mathrm{S}$ wave velocity was fixed equal to $3 \mathrm{~km} / \mathrm{s}$ for Campi Flegrei $(P 1)$ and $2.4 \mathrm{~km} / \mathrm{s}$ for Mt. Vesuvius (Lomax et al., 2001): Medium density $\rho_{0}$ was considered equal to $2.2 \mathrm{gm} / \mathrm{cm}^{3}$ for Campi Flegrei $(P 1)$ and 2.5 $\mathrm{gm} / \mathrm{cm}^{3}$ for Mt. Vesuvius (Zollo et al., 2002).

The radiation pattern term $Y_{\theta \phi}{ }^{S}$ is fixed equal to 1 for both the areas in order to take into account the effects due to the maximum radiated energy. Free surface operator $F$ is set equal to 2 .

High frequency spectral decay $\gamma$ is considered equal for the two areas (table I). This hypothesis is validated by performing a check on Mt. Vesuvius data using the procedure for the evaluation of spectral parameters used for Campi Flegrei, as described in Section 2.2.

The parameters displayed in table I, are used for the evaluation of the PGA values for Campi Flegrei and Mt. Vesuvius. We apply the two techniques described before, making 50 simulations for the second method (GMG). The results displayed in table II show a clear agreement between GMG and RVT methods for both the areas. PGA is equal to $0.04 \mathrm{~g}$ (GMG, RVT) for Campi Flegrei. PGA is equal to $0.10 \mathrm{~g}$ (RVT) and $0.14 \mathrm{~g}$ (GMG) for Mt Vesuvius.

\section{Discussion and conclusions}

In this paper PGA at Campi Flegrei and Mt. Vesuvius was calculated using two techniques based on the stochastic approach. The knowledge of the scaling law of the seismic spectrum, calculated for earthquakes with low-to-moderate magnitude, allowed an extrapolated estimate of the source parameters for earthquake with the maximum expected magnitude. Distance-dependence correction and attenuation effects were taken into account for the definition of the amplitude spectrum of the ground motion. Site effects that generally mask the effective frequency content of the source spectrum were estimated to be negligible and thus have been not considered in the numerical simulation and for the evaluation of ground motion parameters.

We estimate the values of PGA using the parameters shown in table $\mathrm{I}$. The results show a general agreement between RVT and GMG methods for the two areas (see table II). For Campi Flegrei area the maximum expected acceleration obtained in this work is lower (at least by a factor around 2) than the expected PGA due to strong earthquakes with epicenter in the Apennines, whilst for Mt. Vesuvius the two values are comparable (see the map of PGA for Italy, available on line at www.mi.ingv.it). The difference between the PGA values found for the two areas essentially depends on the estimated parameters reported in table I and by the different behavior of the scaling law for the local earthquakes in the two areas.

In the present paper an estimate of errors on the PGA values was calculated with the GMG method. The value of uncertainty in $M_{0}\left(M_{\max }\right)$ and in $f_{c}$ are the main sources of uncertainties on PGA. Seismic moment $M_{0}$ was derived using $M_{\max }$ which is affected by an error of $5 \%$ for Campi Flegrei and 3\% for Mt. Vesuvius. These errors combined with the uncertainties on the parameters of eqs. (4.4) and (4.5) give an error on the value of $M_{0}\left(M_{\max }\right)$ of $22 \%$ for Campi Flegrei and $16 \%$ for Mt. Vesuvius. The relative errors on $f_{c}$ parameter are estimated in the order of $7 \%$ for Campi Flegrei and $10 \%$ for Mt. Vesuvius. Taking these errors into account we estimate the uncertainties on the PGA final values. By using GMG method we obtain PGA equal to $0.04 \pm$ $\pm 0.01 \mathrm{~g}$ for Campi Flegrei and equal to $0.14 \pm$ $\pm 0.05 \mathrm{~g}$ for Mt. Vesuvius. These results are in agreement, inside the error bars, with the PGA values (see table II) evaluated with the RVT method. Further details on the evaluation of statistical errors are explained in Appendix. The final results are also influenced by the limitations 
of the methods, which do not include any phase effect due to the propagation rupture and are based on point source models. Despite the several sources of errors, the stochastic simulations are powerful and simple methods to obtain a fast estimate of ground motion parameters in the investigated areas, which can be useful for the definition of the seismic risk.

\section{Acknowledgements}

We are grateful to Gilberto Saccorotti, who provided the source location of earthquakes shown in fig. 3b, and Laboratory of I.P.F. of V.O. for providing basis maps used in fig. 1a-c. Two anonymous reviewers greatly improved the quality of the first version of the present paper.

Appendix. Evaluation of uncertainties on the PGA calculated by GMG method

Following the Gutenberg-Richter relationship the maximum magnitude $M_{\max }$ is equal to

$$
M_{\max }(a, b)=\frac{a}{b} .
$$

Considering the errors $\delta a$ and $\delta b$ on $a$ and $b$ values respectively, the error $\delta M_{\max }$ is given by

$$
\delta M_{\max }=\sqrt{\left(\frac{\partial M_{\max }}{\partial a}\right)^{2}(\delta a)^{2}+\left(\frac{\partial M_{\max }^{2 t}}{\partial b}\right)^{2}(\delta b)^{2}} .
$$

The seismic moment $M_{0}$ is related to the maximum magnitude $M_{\max }$ according to the equation

$$
M_{0}\left(c, d, M_{\max }\right)=10^{\left(c+d M_{\max }\right)} .
$$

Using this relationship the error $\delta M_{0}$ on the seismic moment can be evaluated

$$
\delta M_{0}=\sqrt{\left(\frac{\partial M_{0}}{\partial M_{\max }}\right)^{2}\left(M_{\max }\right)^{2}+\left(\frac{\partial M_{0}}{\partial c}\right)^{2}(\delta c)^{2}+\left(\frac{\partial M_{0}}{\partial d}\right)^{2}(\delta d)^{2}}
$$

We assume that $\delta M_{0}$ and $\delta f_{c}$ are the main sources of error on the evaluation of $\delta a_{\mathrm{rms}}$. If we consider relation (4.1)

$$
a_{\mathrm{mms}}=\sqrt{2 f_{c} \int_{f_{\text {int }}}^{f_{\text {fut }}}\left[a\left(f,\left\{K_{i}\right\}\right)\right]^{2} d f} .
$$

The error $\delta a_{\text {rms }}$ is equal

$$
\delta a_{\mathrm{mms}}=\sqrt{\left(\frac{\partial a_{\mathrm{mis}}}{\partial f_{\mathrm{c}}}\right)^{2}\left(\delta f_{\mathrm{c}}\right)^{2}+\left(\frac{\partial a_{\mathrm{ms}}}{\partial M_{0}}\right)^{2}\left(\delta M_{0}\right)^{2}}
$$

The PGA value is evaluated by considering the maximum value of Gaussian distribution with $\sigma$ equal to $a_{\mathrm{rms}}$. The error on the value of PGA is evaluated by considering the error on the value of $a_{\mathrm{rms}}$ according to

$$
\delta P G A=\sqrt{\left(\frac{\partial P G A}{\partial a_{\mathrm{mss}}}\right)^{2}\left(\delta a_{\mathrm{mss}}\right)^{2}}
$$




\section{REFERENCES}

ABERCROMBIE, R.E. (1995): Earthquake source scaling relationship from -1 to $5 M_{L}$ using seismogram recorded at 2.5-km depth., J. Geophys. Res., 100 (B12), 24,015$24,036$.

AKI, K. (1967): Scaling law of the seismic spectrum, Bull. Seismol. Soc. Am., 72, 1217-1231.

AKI, K. (1986): Physical theory of earthquakes, in Procedings of Strasbourg, 1986 Summer School on Seismic Hazard in Mediterranean Regions.

Akinci, A., L. Malaginini, R.B. Hermann, N.A. Pino, M. SCOGNAmiglio and H. Eyidogian (2001): High-frequency ground motion in the Erzincan region, Turkey: inferences from small earthquakes, Bull. Seismol. Soc. Am., 91, 1446-1455.

ANDERSON, J. and S. Houcin (1984): A model for the shape of Fourier amplitude spectrum of acceleration at high frequencics, Bull. Seismol. Soc. Am. 74, 1969-1993.

Andronico, D., G. Calderoni, R. Cioni, A. Sbrana, R. Supplzio and R. SANTACROCE (1995): Geological map of Somma-Vesuvius Volcano, Per. Mineral, 64, 77-78.

Archuleta, R.J., E. Cranswick, C. Mueller and P. SpuDICH (1982): Source parameters of the 1980 Mammoth Lakes, California, earthquake sequence, J. Geophys. Res., 87, 4595-4607.

Aster, R.C., R.P. Meyer, G. De Natale, A. Zollo, M. Martini, E. DEl. Pezzo, R. SCARPa and G. IAnNaccone (1992): Seismic investigation of the Campi Flegrei Caldera, in Volcanic Seismology, Proc. Volcanol. Ser. III (Springler Verlag, New York).

AtKInson, G.M. and I.A. BERESNEV (2002): Ground motions at Memphis and St. Louis from M 7.5-8 earthquakes in the New Madrid scismic zone, Bull. Seismol. Soc. Am., 92, 1015-1024.

Bianco, F., M. Castellano, E. Del. Pezzo and J. Ibanez (1999): Attenuation of the short period seismic waves at Mt. Vesuvius, Italy, Geophys. J. Int., 138, 67-76.

BOATWRIGHr, J. (1980): A spectral theor for circular scismic sources: simple estimates of source dimension, dynamic stress drop and radiated energy, Bull. Seismol. Soc. Am., 71, 69-94.

BOORE, D.M. (1983): Stochastic simulation of high-frequency ground motions based on seismological models of radiated spectra, Bull. Seismol. Soc. Am., 73, 1865-1984.

BOORE, D.M. (2003): Simulation of Ground Motion using the Stochastic Method, Pure Appl. Geophys., 160, 635-676.

BrunE, J.N. (1970): Tectonic stress and seismic shcar waves from earthquakes, J. Geophys. Res., 75, 4997-5009.

CARTwright, D.E. and M.S. Longuet-Higgins (1956): The statistical distribution of the maxima of a random function, Proc. R. Soc. London, 237, 212-232.

Castellano, M., C. Buonocunto, M. Capello and M. LA Rocca (2001): Seismic surveillance of active volcanoes: the Osservatorio Vesuviano Seismic Network (OVSN - Southern Italy) Seismol. Res. Lett., 73,168175.

Castro, R.R., A. Rovel.l, M. Cocco, M. Di Bona and F. PACOR (2001): Stochastic simulation of strong-motion records from the 26 September $\left(M_{w} 6\right)$, Umbria-Marche (Central Italy) earthquake, Bull. Seismol. Soc. Am., 91, 27-39.
DAvison, F. and C. Scholz (1985): Frequency-moment distribution of earthquakes in the Aleutian Arc: a test of the characteristic earthquake model, Bull. Seismol. Soc. Am., 75, 1349-1362.

Die. Pezzo, E., G. De Natale, G. Scarciella and A. ZolLO (1985): $Q_{C}$ of three-component scismograms of vol. canic microearthquakes at Campi Flegrei volcanic area - Southern Italy, Pure Appl. Geophys., 123, 683-696.

Dei. Pezzo, E., G. De Natale, M. Martini and A. Zollo (1987): Source parameters of microcarthquakes at Campi Flegrei (Southern Italy) volcanic area, Phys. Earth Planet. Inter., 47, 25-42.

Del. Pezzo, E., S. De Martino, M.T. Parrinillo and C. SABBARESE (1993): Seismic site amplification factors in Campi Flegrei, Southern Italy, Phys. Earth Planet. Inter., 78, 106-117.

Del. Pezzo, E., F. Blanco and G. Saccorottr (2003): Seismic source dynamics at Vesuvius Volcano, Italy, $J$. Vol. Geoth. Res. (in press).

De Natal.E, G. and A. Zollo (1986): Statistical analysis and clustcring features of the Phlegrean Fields earthquake sequence (May 1983-May 1984), Bull. Seismol. Soc. Am., 76 (3), 801-814.

GELLER, R.J. (1976): Scaling relation for earthquake source parameters and magnitude, Bull. Seismol. Soc. Am., 66 (5), 1501-1523.

Hanks, T.C. (1979): $b$ values and $\omega^{-k}$ seismic source models: Implications for tectonic stress variations along active crustal fault zones and the estimation of high frequency strong ground motion, J. Geophys. Res., 84, 2235-2242.

Hanks, T.C. (1982): $f_{\max }$ Bull. Seismol. Soc. Am., 72, $1867-$ 1880.

Hiramatsu, Y., H. Yamanaka, K. Tadokoro, K. NishigaMI and S. OHMI (2002): Scaling law between corner frequency and seismic moment of microearthquakes: is the breakdown of the cube law a nature of carthquakes?, Geophys. Res. Lett., 29 (8).

KeIL.IS-BoroK, V.I. (1959): On estimation of the displacement in an earthquake source dimension, Ann. Geofis., 12, 205-214.

LAY, T. and T.C. WALlace (1995): Moder Global Seismology (Academic Press, London).

LomaX, A., A. Zollo, P. Capuano and J. Virieux (2001): Precise, absolute carthquake location under SommaVesuvius volcano using a new 3D velocity model, Geophys. J. Int., 146, 313-321.

MADARIAGA, R. (1976): Dynamics of an expanding circular fault., Bull. Seismol. Soc. Am., 66, 639-666.

PAPAGEORGIU, A.S. (2003): The barrier model and strong ground motion, Pure Appl. Geophys., 160, 603-634.

PoweL, L.A. (1983): Engineering description of the U.W. portable digital seismograph, in Proceedings of the Workshop on Portable Digital Seismograph Development, IASPEI Meeting, Los Altos, CA.

Rosi, M., R. Santacroce and A. Sbrana (1987): Geological map of the Somma-Vesuvius volcanic complex (1:25000), CNR, Progetto Finalizzato Geodinamica, Salomone, Roma.

Saccorotrt, G., F. Bianco, M. Castellano and E. Del PezZo (2001): The July-August 2000 seismic swarms at Campi Flegrei volcanic complex, Italy, Geophys. Res. Lett. 
SANTACROCE, R. (1983): A general model for the behaviour of the Somma-Vesuvius volcanic complex, J. Volcanol. Geotherm. Res., 17, 237-248.

ShoLZ, C. (1968): The frequency-magnitude relation of microfracturing in rock and its relation to earthquakes. Bull. Seismol. Soc. Am., 58, 399-415.

SLfjKo, D., L. PeruzzA and A. Rebez (1998): Seismic hazard maps of Italy., Ann. Geofis., 41 (2), 183-214.
ZOLlo, A., W. MarzocChI, P. CaPuano, A. LomaX and G. IANNACCONE (2002): Space and time behaviour of seismic activity at Mt. Vesuvius Volcano, Southern Italy, Bull. Seismol. Soc. Am., 92, 625-640.

(received March 25, 2004; accepted July 20, 2004) 